\title{
RESEARCH \& DEVELOPMENT OF A VARIABLE POLARISATION SUPERCONDUCTING UNDULATOR AT THE NSLS
}

\author{
Shailendra Chouhan ${ }^{\dagger}$, D. Harder, G. Rakowsky, J. Skaritka, T. Tanabe \\ NSLS, Brookhaven National Lab, Upton, NY 11973, U.S.A.
}

\section{Abstract}

In this paper a new concept for a planar, superconductive, variable polarization undulator (VPU) is presented. Advantage of this design include: (1) electrical tunability for both right and left circular and elliptical, as well as linear vertical or horizontal, (2) it requires no compensation of unwanted vertical field component and (3) used only simple windings of superconductive wire in an interlaced pattern. The construction of the device is described and compared with a permanent magnet VPU with the same gap and period, as well as with previously published concepts.

\section{INTRODUCTION}

There is a growing interest within the synchrotron radiation community in producing high brightness hard $\mathrm{x}$ ray beams in medium-energy $(\sim 3 \mathrm{GeV})$ machines by use of superconducting technology. Planar superconducting undulators (SCU's) are in development at several laboratories and are already beginning commercial production [1].

Recently proposed elliptical polarization SCU concepts, such as the Sasaki "snake-wound" design [2,3], generate either left or right helical fields and produce only one mode of circular or elliptical polarization. Planar-helical SCU concepts by Rossmanith [4] and Hwang [5] provide fully variable polarization, but require superposition of an additional planar undulator field to compensate an inherent vertical field component [4].

This paper describes a new concept to generate variable polarized synchrotron radiation, with electrical tunability for both right and left circular and elliptical, as well as linear vertical or horizontal polarization.

\section{MECHANISM FOR PRODUCING AN ELLIPTICAL FIELD}

When superconductors in the slots of semi cylindrical yokes of low carbon steel placed above and below the beam axis are parallel to each other and perpendicular to the longitudinal axis as in Figure 1, and with alternating current directions as shown, the magnetic fields in the gap are described by the well-known planar undulator equations:

$$
\begin{aligned}
& B_{y}=B_{y 0} \operatorname{Cosh}\left(k_{y} y\right) \operatorname{Cosh}\left(k_{x} x\right) \operatorname{Sin}(k z), \\
& B_{x}=\frac{k_{x}}{k_{y}} B_{y 0} \operatorname{Sinh}\left(k_{y} y\right) \operatorname{Sinh}\left(k_{x} x\right) \operatorname{Sin}(k z),
\end{aligned}
$$

$$
B_{z}=-\frac{k}{k_{y}} B_{y 0} \operatorname{Sinh}\left(k_{y} y\right) \operatorname{Cosh}\left(k_{x} x\right) \operatorname{Cos}(k z),
$$

with the coefficients related by $k_{x}^{2}+k_{y}^{2}=k^{2}$, $k=2 \pi / \lambda_{u}$ and where $\lambda_{u}$ is the undulator period, and $B_{y 0}$ represents the on-axis magnitude of vertical magnetic field. $B_{y 0}$ depends on current density and coil cross-section area, gap-to-period ratio, the magnetic properties of the pole material, and the details of the geometry.

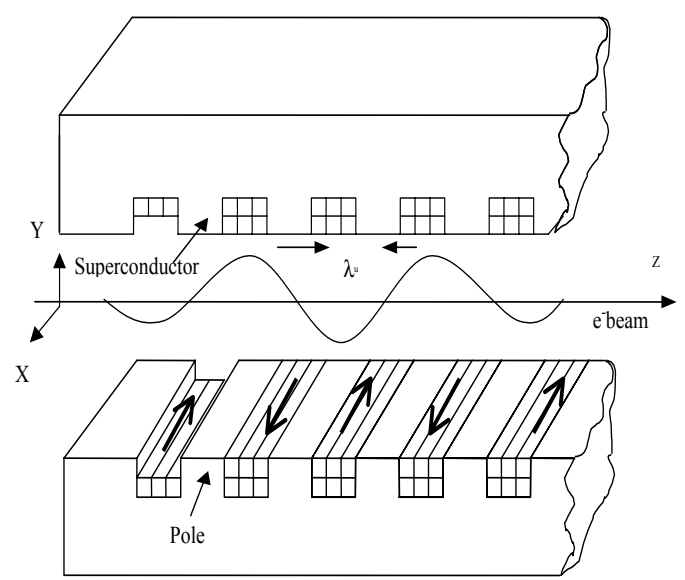

Figure 1: Schematic drawing of a planar sc undulator.

In this case, at the mid plane along the beam axis $(x=y=0)$, only a (nearly) sinusoidal vertical field distribution $B_{y}(z)$ is observable.

But if the conductors are not perpendicular but are inclined by an angle $+\theta$ in the upper yoke and by $-\theta$ in the lower yoke (like Coils A in Figure 2), then both transverse $B_{x}(z)$ and longitudinal components $B_{z}(z)$ are generated along with sinusoidal vertical field $B_{y}(z)$.

Horizontal and vertical components are out of phase, resulting in a helical field. The on-axis helical field from the tilted conductor in the arrays is expressed roughly by

$$
\begin{aligned}
& B_{y}(z)=B_{y 0} \operatorname{Cos}(\theta) \operatorname{Sin}\left(2 \pi z / \lambda_{u}\right), \\
& B_{x}(z)=B_{r e s} \operatorname{Sin}(\theta) \operatorname{Sin}\left(2 \pi z / \lambda_{u} \pm \pi / 2\right) .
\end{aligned}
$$

The magnitude of the longitudinal $B_{z}$ component is zero in the mid-plane.

A second set of conductors (Coils B in Figure 2) inclined by $-\theta$ in the upper yoke and $+\theta$ in the lower yoke, 
also generates a helical field, but of opposite helicity. By connecting each set of coils to a separate bipolar power supply, and varying the currents, all possible polarization modes may be obtained: right or left circular, linear horizontal, linear vertical, or any degree of elliptical polarization. The current density ratio and direction of current in the two coils for different polarization modes from the present model is shown in Table 1.

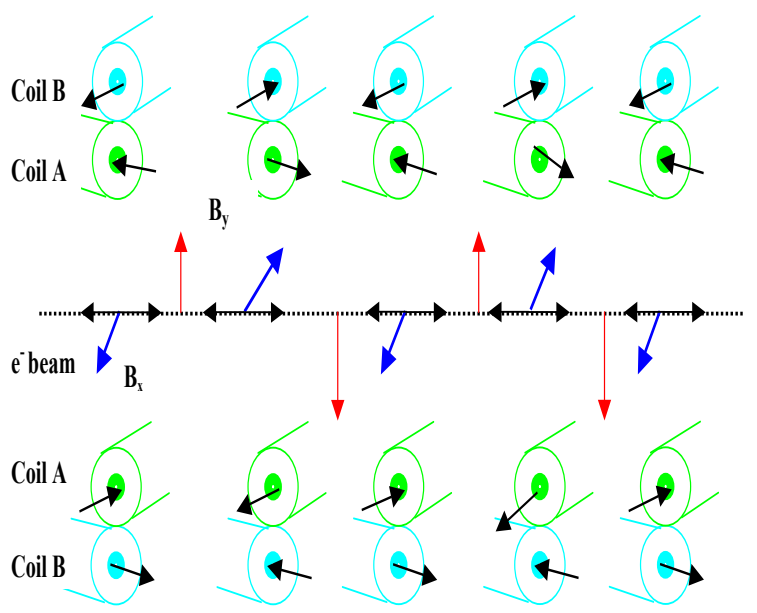

Figure 2: Schematic drawing of a planar variable polarized undulator (VPU).

We use RADIA software package to build a model of the VPU based on above mention concept and calculate expected fields. Figure 3 shows the different modes of field distribution generated on-axis by the device. A period length of $26 \mathrm{~mm}$ and magnetic gap of $6.5 \mathrm{~mm}$ was assumed for the field calculation. It is a multilayer coil with the assumption of 10 turns per layer and a $30^{\circ}$ inclination angle. The current density was assumed to be $1500 \mathrm{~A} / \mathrm{mm}^{2}$ and the peak field achieved with these parameters is 1.0 Tesla.

Table 1: Current Density Ratio and Direction of Current in Coil A and Coil B for Different Polarization Mode

\begin{tabular}{|l|l|l|}
\hline Polarization Mode & $\begin{array}{l}\text { Current Density } \\
\text { Ratio }=\mathrm{J}_{\mathrm{A}} / \mathrm{J}_{\mathrm{B}}\end{array}$ & $\begin{array}{l}\text { Direction } \\
\text { of Current }\end{array}$ \\
\hline R-H Circular & 0.473 & Opposite \\
\hline L-H Circular & 1.642 & Opposite \\
\hline Linear Vertical & 0.921 & Same \\
\hline Linear Horizontal & 0.882 & Opposite \\
\hline
\end{tabular}

In Figure 4, ratio of current density in two coils and the corresponding circular polarized magnetic flux density from conductor at different winding angles $(\theta)$ is examined. We calculate magnetic flux density for constant period-gap ratio while width-height ratio of coil varies with winding angle.
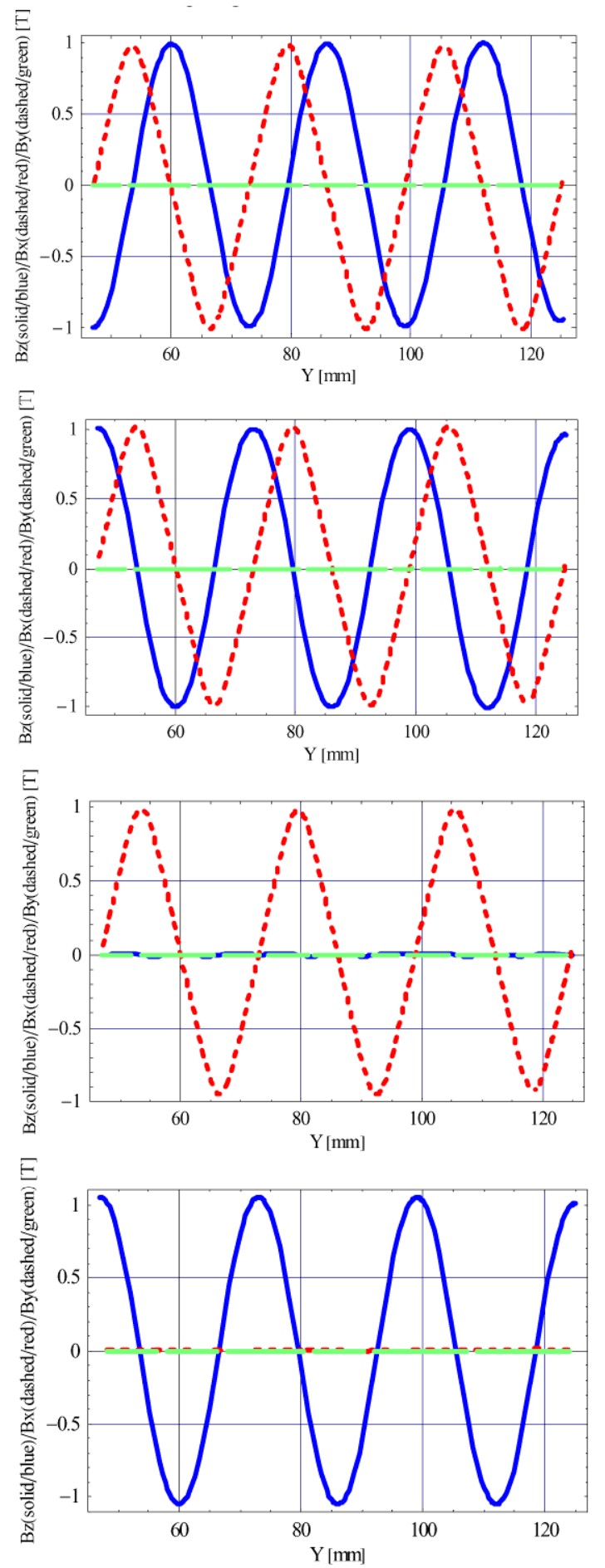

Figure 3: Four different modes of magnetic field distribution in a $26 \mathrm{~mm}$ period $(6.5 \mathrm{~mm}$ minimum gap) planar variable polarized sc undulator. 


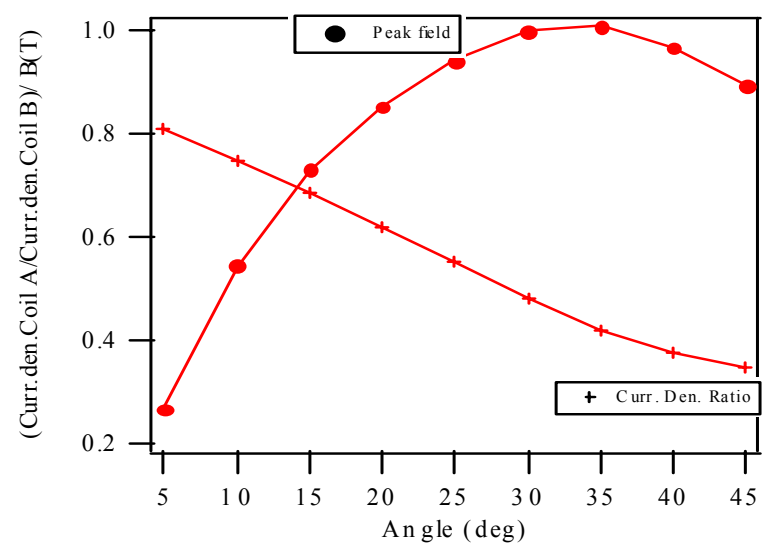

Figure 4: Circularly polarized magnetic flux density for conductors at different winding angles.

Figure 5 shows the list plot of peak field values $\left(B(T)=B_{x 0}(T)=B_{y 0}(T)\right)$ at the minimum gap $(8 \mathrm{~mm})$, corresponding fundamental photon energy $E_{p h}(\mathrm{keV})$ and undulator parameter $(K)$ at the given period length $\lambda_{u}(\mathrm{~mm})$.

Where $K=\sqrt{\left(K_{x}^{2}+K_{y}^{2}\right)}$,

$$
E_{p h}(k e V)=0.95 n E^{2} /\left[\lambda_{u}(\mathrm{~cm})\left(1+K^{2} / 2\right)\right] ;
$$

$n=1$ for fundamental photon energy \& $E(\mathrm{GeV})=$ energy of electron beam.

The field at the mid plane, generated by the device is significantly larger than the field produced by an Appletype device or from previously published concepts [2,3]. Therefore, it has clear impact on photon brightness and photon energy tunability. This device could be a stronger tool for the user, having interest in the variable polarized radiation.

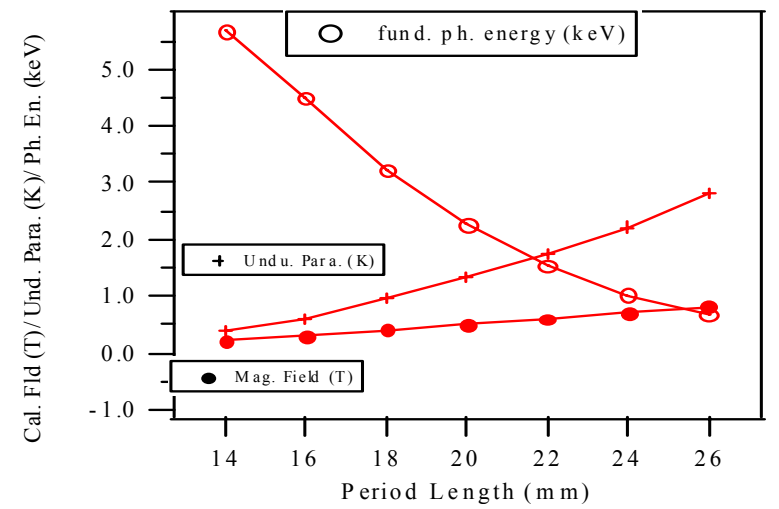

Figure 5: Peak field B (T) at the minimum gap of $8 \mathrm{~mm}$, the undulator deflection parameter $\mathrm{K}$ and the achievable fundamental photon energy $\mathrm{E}_{\mathrm{ph}}(\mathrm{keV})$ for the different period length.

\section{CONCLUSION}

This proposed novel device has the capability to generate not only circularly/ elliptically polarized radiation, but also linear vertically/ horizontally polarized radiation, which may give synchrotron radiation users an opportunity to perform new types of experiments.

\section{NOTICE}

This manuscript has been authored by the Brookhaven Science Associates, LLC under contract number DEAC02-98CH1-886 with the U.S. Department of Energy. The United States Government retains, and the publisher, by accepting the article for publication, acknowledges, a worldwide license to publish or reproduce the published form of this manuscript, or allow others to do so, for the United States Government Purposes.

\section{REFERENCES}

[1] Kubsky et al, "Superconductive mini-gap undulator new way to high-energy photons: latest news," Sync. Radiation Instrumentation Conf. Proc. 2004.

[2] R.P. Walker, "New concept for a planar helical undulator," ELETTRA internal report, Oct.2000 unpublished.

[3] S. Sasaki "Design of a superconducting planar helical undulator, Proc. Workshop on Superconductive Wigglers and Undulators," ESRF, June 2003.

[4] R. Rossmanith et al., "Superconductive undulators with variable polarization direction," Proc. Asian Particle Acce. Copnf. APAC 2004.

[5] C.S. Hwang et al, "Superconducting undulator with variably polarized light," Proc. of the Free Electron Laser Conference, 501-503, 2004. 